\title{
Emerging highlights from novel human coronavirus and angiotensin-converting enzyme 2: promising way to target brain vascular diseases
}

\begin{abstract}
A specific metallopeptidase called angiotensin-converting enzyme 2 (ACE2) has been identified as the modulating receptor on the surface of the endothelium and other human cells infected by the new coronavirus causing Severe Acute Respiratory Syndrome (SARS-CoV-2) and Human Coronavirus Disease 2019 (COVID -19). This modulation of the expression of ACE2 in human cells may be responsible for the production of proinflammatory response with the development of the state of the systemic response of the inflammatory system, hypercoagulability/stasis also an increased risk of both ischemic and hemorrhagic strokes. Therefore, like ACE2, despite being present in almost all human organs, its expression is variable and probably dependent on epigenetic polymorphism, then this is still to be better understood. However, this highlights the importance to understand its pathogenesis and open the doors for the development of future treatment strategies aimed at various diseases related to ACE2, mainly cerebral vascular diseases, and perhaps COVID-19 itself.
\end{abstract}

Keywords: virus biology, angiotensin-converting enzyme 2, cerebrovascular diseases, coronavírus, stroke
Volume 10 Issue 4 - 2020

\author{
Patricia Bozzetto Ambrosi,' Caroline \\ Bozzetto Ambrosi ${ }^{2}$ \\ 'Neuroradiologist, Specialist in Neurology, Neurosurgery and \\ Imaging, Independent Consultant and Academic Researcher, \\ France \\ ${ }^{2}$ Specialist in Internal \& Palliative Medicine and Resident in \\ Cardiology, Brazil
}

\begin{abstract}
Correspondence: Prof. Dr. Patricia Bozzetto Ambrosi, Neuroscientist, Neuroradiologist and Neurological Surgeon, Independent Consultant and Academic Researcher, France, Email patriciabozzetto@outlook.com
\end{abstract}

Received: May 28, 2020 | Published: July 09, 2020

\section{Introduction}

In late 2019, in the Chinese city of Wuhan, a new coronavirus was identified as the cause of a set of atypical pneumonia cases. ${ }^{1}$ A few days later, an epidemic was declared when similar cases spread rapidly across China and other countries, leading to the globalization of the pandemic. ${ }^{2}$ Three months later, the World Health Organization designated the disease as Human Coronavirus Disease 2019 (COVID-19) and the virus as coronavirus for the severe acute respiratory syndrome (SARS-CoV-2). ${ }^{2,3}$

Despite there is still no complete information on the natural history or neither complete measures of unquestionable effectiveness for the clinical management of cases of human SARS-CoV-2 infection, with many details to be determined. ${ }^{4,5}$ There has been observed that the spectrum of the clinical presentation of COVID-19 varies from mild to critical, with most infections not being serious; however, serious and critical cases represent about 5 to $10 \%$ of cases. These cases are manifested by respiratory failure with increased production of pro-inflammatory cytokines, leading to an exacerbated systemic inflammatory response, multiple organ dysfunction, and a very serious condition: the systemic inflammatory response syndrome (SIRS), hypercoagulability/stasis and shock. ${ }^{5,6}$

In addition, an increased risk of cerebrovascular events has been reported with SARS-CoV-2 infection and COVID-19, including hemorrhagic and ischemic strokes related to an increased risk of stasis and thrombocytic state of vessels. ${ }^{7}$ Case report series have reported an association between major occlusions of the arterial circle of Willis vessels and COVID-19, particularly in younger patients with catastrophic and malignant evolution as illustrated in Figure 1. Also given the complex thrombocytic pathogenesis of COVID-19related abnormalities, this also refers to the lack of evidence-based standardization of treatments including stroke and other vascular lesions ensuring that treatments are not overshadowed by the pandemic. ${ }^{8,9}$ In the same time, there is a need for rethinking protocols of treatment as much endovascular treatment related to use of stents and other devices that are directly related to clot formation and change of blood rheology.

Interestingly, in the midst of this emerging epidemy, strain studies have shown that coronaviruses exhibit a tendency to bind cells to specific receptors, particularly ACE2. ${ }^{10}$ This enzyme regulates the renin-angiotensin-aldosterone system (RAAS), controlling and modulating endothelial cells in blood vessels and various organs. Therefore, it is not surprising that initial reports have suggested that diseases related to SIRS, such as hypertension, diabetes, cardiovascular diseases, and even stroke, are the most frequent comorbidities in COVID-19 disease. ${ }^{10,11}$

It is also important to note that this may allow a better understanding of the basis of some serious complications, such as extrapulmonary, including brain vascular insults/stroke events. The fact that ACE2 is an important enzyme component of most human organs and at the same time acts at the main point of the virus domain. It is still possible that the polymorphism of the ACE2 gene, the expression of the human ACE2 mRNA, the polymorphism of the human ACE2 protein influence the susceptibility to SARS-CoV-2 and the result of the disease by COVID- 19 .

\section{ACE2 expression}

The structure of the ACE2 contains a zinc-metallopeptidase enzyme and shows two main domains: the N-terminalpeptidase M2 domain and a C-terminal colectrin renal amino acid transporter. ${ }^{11,12}$ The extracellular domain of ACE2 is cleaved from the transmembrane domain by another enzyme known as sheddase, and the resulting 
soluble protein is released into the bloodstream and finally excreted in the urine. It is responsible for the conversion of angiotensin II to angiotensin 1-7. ACE2 is an important enzyme component of RAAR that is present in most human organs ACE2 is linked to the cell membrane mainly of the alveolar epithelial cells of the lung, enterocytes of the small intestine, arterial and venous endothelial cells, and arterial smooth muscle cells in most organs as represented in Figure 2. ${ }^{11}$

The ACE2 converts angiotensin II to angiotensin (1-7) and therefore can protect against the effects of angiotensin II. The apelin receptors (particularly Apelin-12/13) that are widely distributed in the cardiovascular system, and are closely involved in the pathogenesis of hypertension, heart disease, and atherosclerosis, and consequently cerebrovascular diseases. ${ }^{13}$ The ACE2 and Apelin genes are located on the $\mathrm{X}$ chromosome. Apelin peptides, as important pharmacological targets, mediate crucial physiological effects on the vasculature of the heart and vessels, while blocking Ang II signaling improves cardiac function and reduces hypertension and related cardiovascular events in patients. On the other hand, the peptide pyr1-apelin-13 negatively regulates cardiac remodeling mediated by Ang II, fibrosis injury. The improvement in cardiac dysfunction and injury and hypotensive effects of pyr1-Apelin-13 in ApoE KO mice are probably driven by increased levels of ACE2 and activation of protective signaling transduction pathways, including phosphorylation of Akt / ERK1 / 2 and eNOS. ${ }^{14,15}$

ACE2 deficiency leads to hypertension and impaired heart function, while recombinant ACE2 is protective against heart and vascular disease. The serum activity of ACE2 is greater in patients with hypertension or heart failure compared to healthy individuals, while plasma levels of apelin are suppressed in patients with heart disease. The apelin and ACE2 pathways represent candidate therapeutic targets for the treatment of hypertension, atherosclerosis, and heart failure in preclinical and in vitro models. ${ }^{15}$

\section{COVID-I9 and ACE2 polymorphism expression in human tissues}

Researchers have discovered, through complete genome sequencing and phylogenetic analysis, that the cause of COVID-19 is a beta-coronavirus in the same subgenus as the SIRS (in addition to several bat coronaviruses), but in a zoonotic type different. In a phylogenetic analysis of 103 strains of SARS-CoV-2 from China, two different types of SARS-CoV-2 were identified, designated type L (70\% of strains) and type S (representing 30\%). Type L predominated in the early days of the epidemic in China, but it represented a lower proportion of strains outside Wuhan than in Hubei province. ${ }^{16,17}$

To date, there are reports from various groups on mutations observed in strains from different countries. When phylogenetically analyzing the 2019 coronavirus network. Researches from Cambridge, UK found that when analyzing 160 complete genomes of SARS$\mathrm{CoV}-2$ and found that there are three central variants, differentiated by protein changes, which he called A, B, and C. Type A looks like to be ancestor of the external group of bats. Types $\mathrm{A}$ and $\mathrm{C}$ are found in significant proportions outside East Asia, that is, in Europeans and Americans. On the other hand, type B is the most common type in East Asia, and its ancestral genome does not appear to have spread outside East Asia without first becoming B-derived types, pointing to founding effects or immunological or environmental resistance against it like outside Asia. ${ }^{18,19}$
The fact that ACE 2 serves as the main point of entry into cells for most coronaviruses, including SARS-CoV-2 (the virus that causes COVID-19). More specifically, the binding of the SARS-CoV2 sp1 S1 protein to the enzymatic domain of ACE2 on the cell surface results in endocytosis and translocation of the virus and enzyme into endosomes located in the cells. This entry process also requires the initiation of protein $\mathrm{S}$ by the host serine protease TMPRSS2, whose inhibition is currently under investigation as a therapeutic potential for COVID-19. ${ }^{20}$

Physiologically, ACE2 reduces blood pressure by catalyzing the cleavage of angiotensin II (vasoconstrictor peptide) into angiotensin (1-7) (vasodilator). ACE2 as a transmembrane enzyme also serves as an entry point into the cells of some coronaviruses, including $\mathrm{HCoV}$ NL63, SARS-CoV (the virus that causes SARS) and SARS-CoV-2. The human version of the enzyme is often referred to as hACE2. It emerged as potent regulators of blood pressure and cardiovascular function, along with the pathways we described above. The increased expression of ACE-2 was also more expressed in some enzymes capable of modifying the functioning of proteins known as histones, which are in the nucleus of cells - linked to DNA - and help to regulate gene expression. This biochemical phenomenon is known as epigenetic modification (when there is a change in the expression pattern of the gene without any change in the DNA). ${ }^{16}$

In view of the knowledge of the involvement of ACE2 in the pathophysiology of coronavirus infection, it is speculated that the modulation of this pathway may be an alternative to be explored in the management of these patients. This has led some to assume that decreasing ACE2 levels in cells can help fight infection. On the other hand, ACE2 has been shown to have a protective effect against virus-induced lung injury, increasing the production of the vasodilator angiotensin 1-7. Furthermore, according to studies carried out in mice, the interaction of the human coronavirus spike protein with ACE2 induces a drop in ACE2 levels in cells through internalization and degradation of the protein and, therefore, may contribute to lung damage. Ciaglia et. al suggested that genetic variants in the ACE2 gene may have a potential to affect the level of ACE2 in the human body and speculated that among different polymorphisms they may exhibit primary effects on ACE2 levels. ${ }^{21,22}$

In the past, recombinant human ACE2 (rhACE2) has been presumed as a new therapy for acute lung injury and appeared to improve pulmonary hemodynamics and oxygen saturation in piglets with a lipopolysaccharide-induced acute respiratory distress syndrome. The half-life of rhACE2 in humans is about 10 hours and the onset of action is 30 minutes, in addition to the effect course (duration) of 24 hours. Several findings suggest that rhACE2 may be a promising drug for those with intolerance to classic RAAR inhibitors or in diseases in which circulating angiotensin II is elevated. Infused rhACE2 has been evaluated in clinical trials for the treatment of acute respiratory distress syndrome. ${ }^{23-26}$ Studies involving the use of ACE inhibitors have been associated with a significant reduction in the risk of pneumonia compared to controls (34\%). In addition, the risk of pneumonia was also reduced in patients treated with ACE inhibitors who were at increased risk of pneumonia, particularly in those with stroke and heart failure. The use of ACE inhibitors has also been associated with a reduction in pneumonia-related mortality, although the results have been less robust than in the overall risk of pneumonia. ${ }^{22,23}$ 


\section{ACE2 activity and brain vascular diseases}

Cerebral vascular diseases include a variety of medical conditions that affect blood vessels in the brain and hemostasis of the circulation. These disorders usually damage or deform the arteries that supply oxygen and nutrients for brain damage. Ischemic insult, or sometimes a hemorrhagic stroke usually caused by a temporary or permanent reduction in cerebral blood flow clinically manifested by occlusions
(Figure 1), is a major cause of mortality and disability in the world. Various pathophysiological conditions, such as hypertension, atherosclerosis and prothrombotic states have been found to increase the risk of ischemic stroke. In addition, high levels of oxidative stress and inflammatory response in the brain in the early stages of ischemic stroke also contribute to irreversible brain damage after the ischemic insult ${ }^{27-29}$
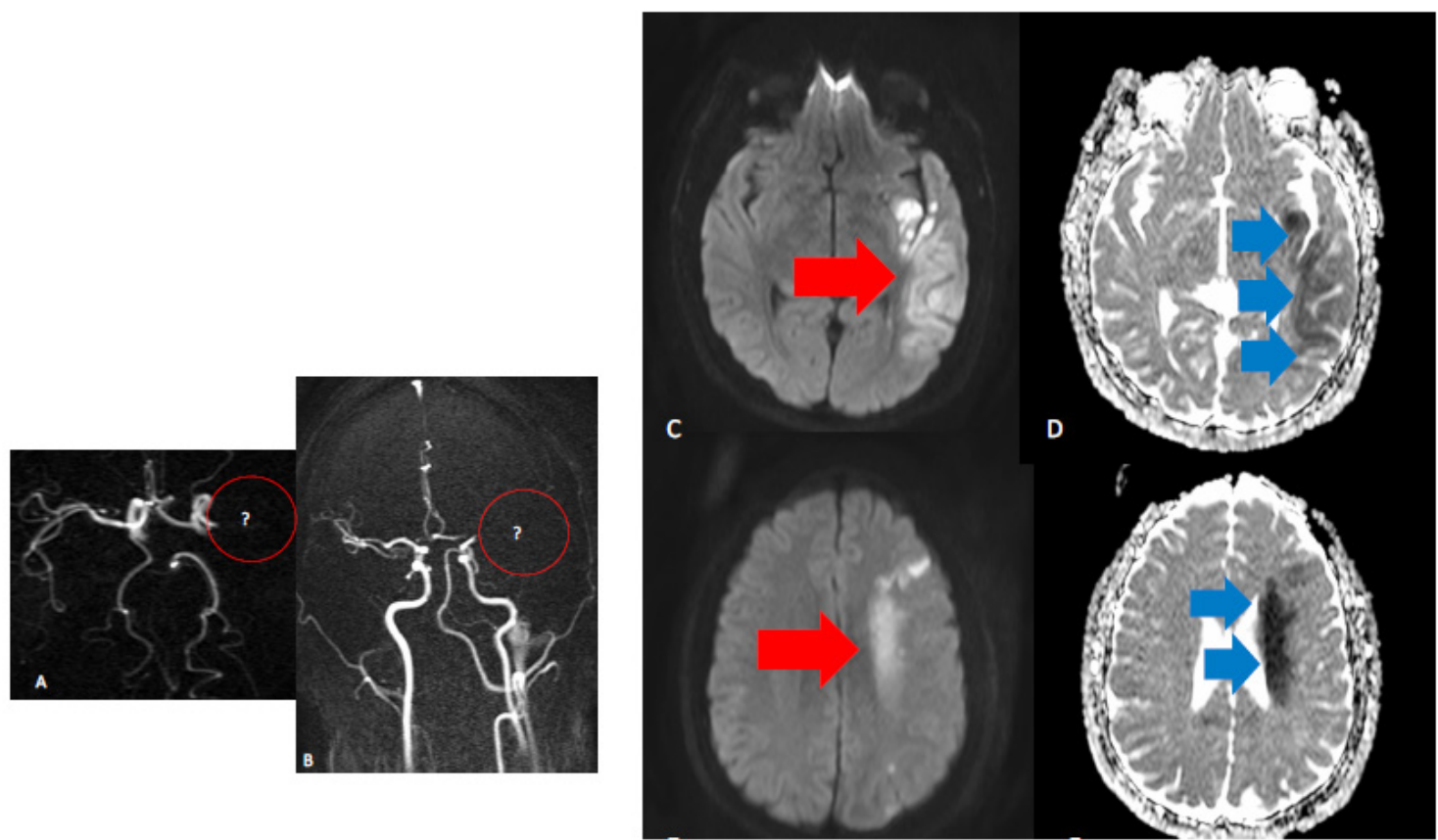

Figure I Left middle cerebral territory appearances in COVID - 19 scenario.A, B occlusion of left MCA on the MRA (see red circles areas) C,D,E,F DWI shine and $A D C$ abnormalities in the territory portions affected pointed by red and blue arrows.

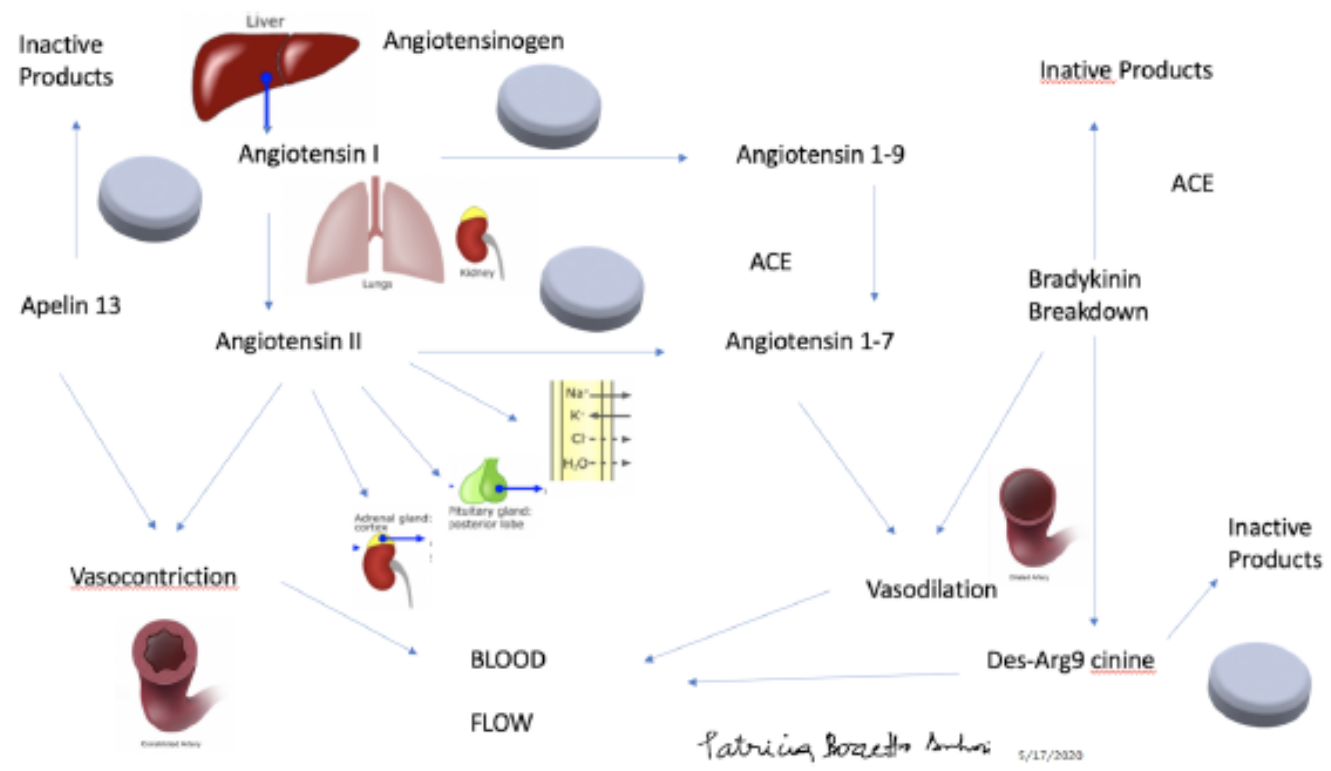

Figure 2 Scheme of action of ACE2 (tablet) in the Renin-Angiotensin-Aldosterone system (RAAS). 
In the brain, the RAAR system acts as a crucial regulator for physiological homeostasis and diseases of the cerebrovascular system, probably related to flow regulation. According to several recent studies, the deleterious effects of the angiotensin-converting enzyme (ACE) receptor axis - angiotensin II (Ang II) angiotensin II (AT1R) of the brain and new components of RRAS, such as ACE2 and angiotensin- (1-7) [Ang - (1-7)], have been identified in the brain and attenuate the development of hypertension, and the pathological progress of atherosclerosis and ischemic stroke. The genetic and pharmacological inhibition of ACE2 increases the plasma levels of pir1-apelin-13 and apelin-17 and increases its vasodepressor action. The angiotensin II produces oxidative stress and endothelial dysfunction in the cerebral arteries, and type I angiotensin II receptors can play a role in longevity and vascular aging. ${ }^{30,31}$

It was even raised the hypothesis that ACE2 deficiency increases oxidative stress and endothelial dysfunction in the cerebral arteries and examined the role of ACE2 in age-related cerebrovascular dysfunction. ACE2 deficiency impaired endothelial function in the cerebral arteries in adult mice and increased endothelial dysfunction during aging. Neuroprotection of the ACE2-Mas axis after insulting ischemia was verified in two different models of cerebral ischemia in rats. In rats with endothelin, -1 transient occlusion of the middle cerebral artery induced by -1 , an ACE2 activator could significantly attenuate the size of cerebral infarction and neurological deficits after transient ischemia. While the same study found that central administration of Ang- (1-7) minimized the size of cerebral infarction and improved neurological functions in a mouse model with permanent middle cerebral artery occlusion. ${ }^{32}$

In the recent past, the discovery of beneficial neuroprotective effects of the angiotensin-converting enzyme 2-angiotensin- (1-7) -But axis [ACE2-Ang- (1-7) ] in ischemic and hemorrhagic stroke has aroused interest in further characterization full of its mechanisms of action. The latter incorporates evidence describing the actions of Ang-(1-7) to combat the deleterious effects of angiotensin II through its type 1 receptor, including anti-inflammatory, antioxidant, vasodilator and angiogenic effects, and the role of altered signaling kinase phosphatase. Mas interactions with other receptors, including bradykinin receptors and angiotensin 2 receptors, should also be considered. ${ }^{32,33}$ The hypothesis has been raised that ACE2 deficiency increases oxidative stress and endothelial dysfunction in the cerebral arteries and examined the role of ACE2 in age-related cerebrovascular dysfunction. ACE2 deficiency impaired endothelial function in the cerebral arteries of adult mice and increased endothelial dysfunction during aging. Oxidative stress plays a critical role in cerebrovascular dysfunction induced by ACE2 deficiency and aging. ${ }^{32,33}$

A recent analysis of single-cell RNA sequencing (RNA-seq) indicated that Asian men may have a higher expression of ACE2 tissue. In another case-control study carried out in the Han population of northeastern China, ACE2 serum activity correlated negatively with body mass index, pulse pressure, and estrogen levels in female patients with essential hypertension. These observations point to a cardiovascular protective effect of circulating ACE2 levels and simultaneously prove that estrogens participate in the positive regulation of ACE2 expression and activity levels. This may explain the relative protection of women versus men in COVID-19 infection. Taken together, this evidence seems to indicate that the sexual predisposition to COVID-19, with men being little more susceptible, may reflect a peculiar plasma profile of ACE. Recent research has suggested that genetic variants in the ACE2 gene may have the potential to affect the level of ACE2 in the human body and speculated that between different polymorphisms they may exhibit primary effects on ACE2 levels. . $^{34,35}$

\section{Final remarks}

Recently ACE2 has been identified as a factor for the main entry point for SARS-CoV-2 that causes COVID-19. As part of the renin-angiotensin system in the brain, it acts as a crucial regulator of physiological homeostasis through flow regulation and endothelial modulation probably related to diseases of the cerebrovascular system, and other risk factors involved in the pathophysiology of stasis and vascular thrombocity. A more complete understanding of the mechanisms of epigenetic modulation involving the pathogenesis of cerebrovascular diseases and the mechanisms that trigger vascular thrombosis and neuroprotection. All of these involved will serve as an essential step in the search for better potential therapies aimed at the clinical scenario of cerebrovascular diseases. In the near future, the development of drugs to treat infections by SARS-CoV-2 and other diseases related to ACE ligands may help to unveil important aspects of the pathophysiology of vascular brain diseases and to develop new strategies for more targeted treatment.

\section{Acknowledgments}

None.

\section{Conflicts of interest}

The author declares no conflicts of interest.

\section{References}

1. World Health Organization (WHO). Director-General's remarks at the media briefing on 2019-nCoV on 11. 2020.

2. World Health Organization (WHO). Pneumonia of unknown cause - China.

3. Coronaviridae Study Group of the International Committee on Taxonomy of VThe species. Severe acute respiratory syndrome-related coronavirus: classifying 2019-nCoV and naming it SARS-CoV-2. Nat Microbiol. 2020;5:536-544.

4. Wang D, Hu B, Hu C, et al. Clinical Characteristics of 138 Hospitalized Patients With 2019 Novel Coronavirus-Infected Pneumonia in Wuhan, China. JAMA. 2020;23(11):1061-1069.

5. Zhou F, Yu T, Du R, et al. Clinical course and risk factors for mortality of adult inpatients with COVID-19 in Wuhan, China: a retrospective cohort study. Lancet. 2020;395(10229):1054.

6. Canrong $\mathrm{Wu}$, Yang Liu, Yueying Yang, et al. Analysis of therapeutic targets for SARS-CoV-2 and discovery of potential drugs by computational methods. Acta Pharmaceutica Sinica. 2020;10(5):766-788.

7. Jin $\mathrm{H}$, Hong $\mathrm{C}$, Chen $\mathrm{S}$, et al. Consensus for prevention and management of coronavirus disease 2019 (COVID-19) for neurologists. Stroke Vasc Neurol. 2020;5(2):146-151.

8. Salahuddin H, Castonguay AC, Zaidi SF, et al. Interventional Stroke Care in the Era of COVID-19. Front Neurol. 2020;11:468. 
9. Oxley TJ, Mocco J, Majidi S, et al. Large-Vessel Stroke as a Presenting Feature of Covid-19 in the Young. $N$ Engl J Med. 2020;82(20):e60

10. Hamming I, Timens W, Bulthuis ML, et al. Tissue distribution of ACE2 protein, the functional receptor for SARS coronavirus. A first step in understanding SARS pathogenesis. $J$ Pathol. 2004;203(2):631.

11. Turner AJ. Chapter 25: ACE2 Cell Biology, Regulation, and Physiological Functions. In: Unger T, Ulrike M, Steckelings $\mathrm{UM}$, dos Santos RA, editors. The Protective Arm of the Renin Angiotensin System (RAS): Functional Aspects and Therapeutic Implications. Academic Press. 2015. pp. 185-189.

12. Nicholls J, Peiris M. Good ACE, bad ACE do battle in lung injury, SARS. Nature Medicine. 2005;11(8):821-822.

13. Zhen-Zhou Zhang, Wang Wang, Hai-Yan Jin, et al. Apelin Is a Negative Regulator of Angiotensin II-Mediated Adverse Myocardial Remodeling and Dysfunction. Hypertension. 2017;70(6):1165-1175.

14. Japp AG, Newby DE. Unlocking the Therapeutic Potential of Apelin.6. Hypertension. 2016;68(2):307-309.

15. Akhmerov Akbarshakh, Marban Eduardo. COVID-19 and the Heart. Circ Res. 2020;126(10):1443-1455.

16. Mohammed Arif, Sadayappan S, Beckjer RC, et al. Epigenetic modification: a regulatory mechanism in essential hypertension. Hypertens Res. 2019;42(8):1099-1113.

17. Tang $\mathrm{X}, \mathrm{Wu} \mathrm{C}, \mathrm{Li} \mathrm{X}$, et $\mathrm{AL}$. On the origin and continuing evolution of SARS-CoV-2. Natl Sci Rev. 2020.

18. https://www.genengnews.com/news/spread-of-earlycoronavirus-lineages-reconstructed-using-genetic-networktechnique

19. Forster Peter, Forster Lucy, Renfrew Colin, et al. Phylogenetic network analysis of SARS-CoV-2 genomes. Proc Natl Acad Sci U S A. 2020;117(17): 9241-9243.

20. Zhou P, Yang XL, Wang XG, et al. A pneumonia outbreak associated with a new coronavirus of probable bat origin. Nature. 2020;579(7798):270.

21. Muthiah Vaduganathan, Orly Vardeny, Thomas Michel, et al. Renin-Angiotensin-Aldosterone System Inhibitors in Patients with Covid-19. N Engl J Med. 2020;382(17):1653-1659.

22. Kai H, Kai M. Interactions of coronaviruses with ACE2, angiotensin II, and RAS inhibitors-lessons from available evidence and insights into COVID-19. Hypertens Res. 2020; 43(7):648-654.
23. Daniel Caldeira, Joana Alarcão, António Vaz-Carneiro, et al. Risk of pneumonia associated with use of angiotensin converting enzyme inhibitors and angiotensin receptor blockers: systematic review and meta-analysis. BMJ. 2012;345:e4260.

24. Keiji Kub, Yumiko Imai, Shuan Rao, et al. Lessons from SARS: control of acute lung failure by the SARS receptor ACE2. J Mol Med (Berl). 2006;84(10):814-820.

25. Haibo Zhang, Andrew Baker. Recombinant human ACE2: acing out angiotensin II in ARDS therapy. Crit Care.2017;21(1):305.

26. Teng Jiang, Li Gao, Jie Lu, et al. Bentham Science Publishers. ACE2-Ang-(1-7)-Mas Axis in Brain: A Potential Target for Prevention and Treatment of Ischemic Stroke. Current Neuropharmacol. 2013;11(2):209-217.

27. Bozzetto Ambrosi P. New Insights into Cerebrovascular Diseases. Intechopen.

28. Cerebrovascular disease - Introduction - NHS Choices". www.nhs.uk. (accessed on 17 May 2020)

29. Veerasingham SJ, Raizada MK. Brain renin-angiotensin system dysfunction in hypertension recent advances and perspectives. Br J Pharmacol. 2003;139(2):191-202.

30. Feng Y, Xia H, Santos RA, et al. Angiotensin-converting enzyme 2 a new target for neurogenic hypertension. Exp Physiol. 2010;95(5):601-606.

31. Teng Jiang, Li Gao, Jie Lu, et al. Bentham Science Publishers. ACE2-Ang-(1-7)-Mas Axis in Brain: A Potential Target for Prevention and Treatment of Ischemic Stroke. Current Neuropharmacol. 2013;11(2):209-217.

32. Jiang T, Gao L, Gun J, et al. Suppressing inflammation by inhibiting NF-?B pathway contributes to the neuroprotection of angiotensin-(1-7) in rats with permanent cerebral ischemia. Br J Pharmacol. 2012;167(7):1520-1532.

33. Mecca AP, Regenhardt RW, O'Connor TE, et al. Cerebroprotection by angiotensin-(1-7) in endothelin-1induced ischaemic stroke. Exp Physiol. 2011;96(10):10841096.

34. G Y Oudit 1, Z Kassiri, C Jiang, et al. SARS-coronavirus modulation of myocardial ACE2 expression and inflammation in patients with SARS. Eur J Clin Inves. 2020;39(7):618-625.

35. Ciaglia E, Vecchione C, Puca A. COVID-19 Infection and Circulating ACE2 Levels: Protective Role in Women and Children. Front Pediatr. 2020;8:206. 